\title{
Influencia del fenómeno El Niño-La Niña y cambio climático en el patrón de lluvias en São Carlos-SP, Brasil
}

\section{INTRODUCCIÓN}

Basado en las observaciones del aumento de la temperatura media global del aire y del océano, en el derretimiento generalizado de nieve y de hielo, y en el aumento global del nivel medio del mar, el último informe del IPCC sobre cambio climático revela que las alteraciones en los patrones climáticos globales son evidentes. Además indica un probable aumento de la frecuencia de precipitaciones intensas en los últimos 50 años, con una tendencia de probable intensificación en el futuro (IPCC, 2007).

Estos cambios globales también se manifiestan en los diferentes parámetros climáticos a escala local. Dufek y Ambrizzi (2008) exponen evidencias de las variaciones climáticas en América del Sur y en Brasil, y analizan estadísticamente el registro pluviográfico de 59 estaciones en el estado de Sao Paulo entre los años de 1950 y 1999, constatando un incremento significativo en la precipitación total en mas de un $45 \%$ de las estaciones analizadas, asociado al aumento en la frecuencia de lluvias intensas y superiores a $20 \mathrm{~mm}$. Un análisis estadístico de las lluvias a largo plazo — 116 años de registro pluviométricoen el municipio de Campinas-SP no encontró variaciones significativas (Blain et al., 2007), concluyendo que los cambios en el patrón de lluvias no es aplicable para el conjunto del estado de Sao Paulo.

Uno de los fenómenos que proporciona alteraciones en el clima de América del Sur es El Niño Oscilación del Sur (ENOS), ocasionado por una alteración del sistema océano-atmósfera, en el océano Pacífico tropical. Su fase cálida, 
conocida como «El Niño», produce una mayor evaporación en el océano, generando mayores precipitaciones en América del Sur. Lo contrario ocurre en la fase fría, llamada «La Niña» (IMPE, 2011). Diversos autores han analizado las anomalías producidas por ENOS sobre las precipitaciones en diferentes regiones de Brasil. En el estado de Sao Paulo se ha constatado un aumento en la frecuencia y en la precipitación total de lluvias en los meses de abril y mayo en los años de incidencia de «El Niño» (Nunes, 2000; Soler et al., 2010).

De esta forma, el presente trabajo tiene como objetivo evaluar el efecto del cambio climático y la influencia del fenómeno ENOS sobre las precipitaciones de los últimos 50 años en el municipio de São-Carlos-SP.

\section{MATERIALES Y MÉTODOS}

Los datos pluviométricos diarios utilizados en el presente estudio proceden de la estación climática ubicada en el municipio de São-Carlos-SP (latitud $21^{\circ}$ $58^{\prime}$ S, longitud $7^{\circ} 52^{\prime} \mathrm{W}, 856 \mathrm{~m}$ de altitud) (figura 1), y han sido facilitados por el Instituto Nacional de Meteorología (INMET).

\section{FIGURA 1}

\section{UBICACIÓN DEL MUNICIPIO DE SÃO CARLOS EN EL ESTADO DE SÃO PAULO, BRASIL}

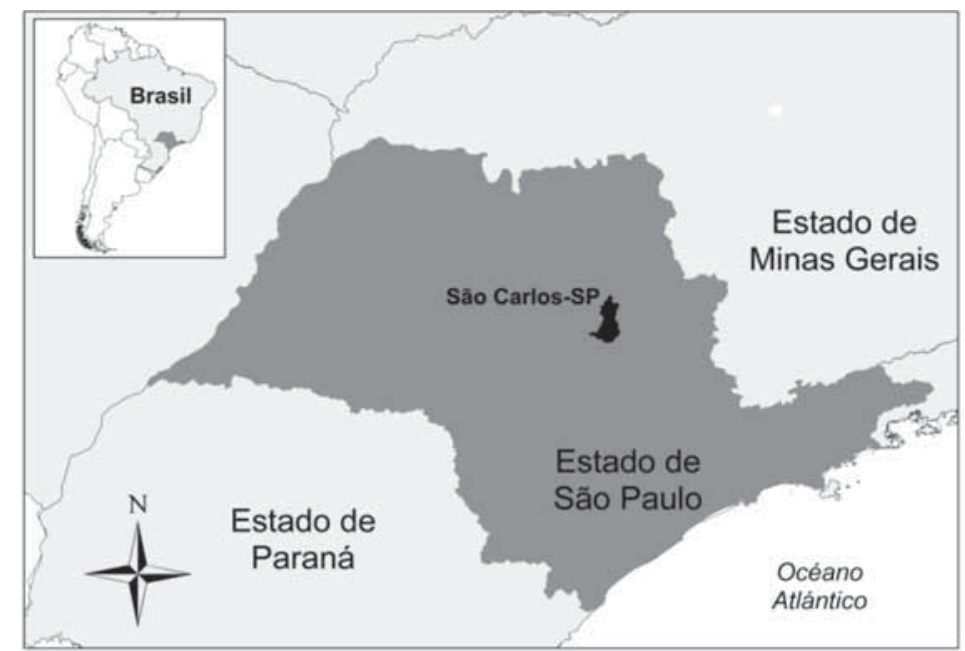

Fuente: elaboración propia.

Estudios Geográficos, Vol. LXXIII, 272, pp. 325-331, enero-junio 2012

ISSN: 0014-1496, eISSN: 1988-8546, doi: 10.3989/estgeogr.201212 
Los datos analizados corresponden al periodo de 01-1961 a 07-2010, siendo excluidos los años 1968, 1985 y 1986 por la ausencia y por las imprecisiones en el registro pluviométrico.

Con los datos disponibles se calculó la precipitación total anual (PPtot), los días de lluvia (Días), la precipitación máxima en 24 horas (PPmax), y el número de lluvias mayores a $20 \mathrm{~mm}$ dia-1 $(>20 \mathrm{~mm})$ para cada año pluviométrico (octubre-septiembre).

Se evaluó la relación entre los parámetros de las precipitaciones indicados previamente mediante el coeficiente de correlación. Los datos anuales fueron agrupados por década para determinar las posibles variaciones causadas por el cambio climático, en tanto que para el fenómeno ENOS se analizó su incidencia según año de fase cálida, fría o neutra (COAPS, 2011). Se estimó la existencia de diferencias estadísticamente significativas $(\mathrm{P}<0,05)$ de estos factores sobre el patrón de lluvias mediante un análisis de varianza unifactorial utilizando el software Minitab 16 (Minitab Inc., Pennsylvania, USA, 2010).

\section{RESUlTADOS}

Los gráficos de las variables pluviométricas anuales evaluadas en el presente estudio, su media por decenio y fase ENOS son presentados en la figura 2.

En el periodo analizado se constató que la mayor precipitación total anual $\left(2.216 \mathrm{~mm}\right.$ ), días de lluvias (164) y precipitaciones mayores a $20 \mathrm{~mm} \mathrm{dia}{ }^{-1}$ (34) se presentaron durante el año 1982, coincidiendo con la ocurrencia de «El Niño». Por el contrario, la menor precipitación $(940 \mathrm{~mm})$ y días de lluvia (81) se presentó dos años antes, en 1980. Al interpretar este comportamiento con el coeficiente de correlación (cuadro 1), es posible afirmar que existe una relación directa y estadísticamente significativa $(\mathrm{P}<0,05)$ entre la precipitación total, eventos mayores a $20 \mathrm{~mm}$ y días de lluvias. De esta forma, en los años lluviosos la frecuencia de precipitaciones y de eventos más intensos es mayor.

$\mathrm{Al}$ analizar las medias de decenios para los diferentes parámetros de las precipitaciones es posible distinguir variaciones ascendentes y descendentes, como por ejemplo la diferencia de $200 \mathrm{~mm}$ en la precipitación total entre los años de 1960 y de 1990 (figura 2 y cuadro 2). Sin embargo, mediante el test de varianza se verificó que no existían diferencias significativas entre las medias de los diferentes parámetros evaluados. Lo anterior indica que en el municipio de São-Carlos-SP no es posible identificar los efectos del cambio climático global en el patrón de precipitaciones local. 
FIGURA 2

PATRÓN DE LLUVIAS EN EL MUNICIPIO DE SÃO CARLOS-SP ENTRE LOS AÑOS 1961 Y 2010. SE INDICA LA FASE DEL FENÓMENO ENOS Y LA MEDIA DE LA DÉCADA PARA CADA PARÁMETRO DE PRECIPITACIONES
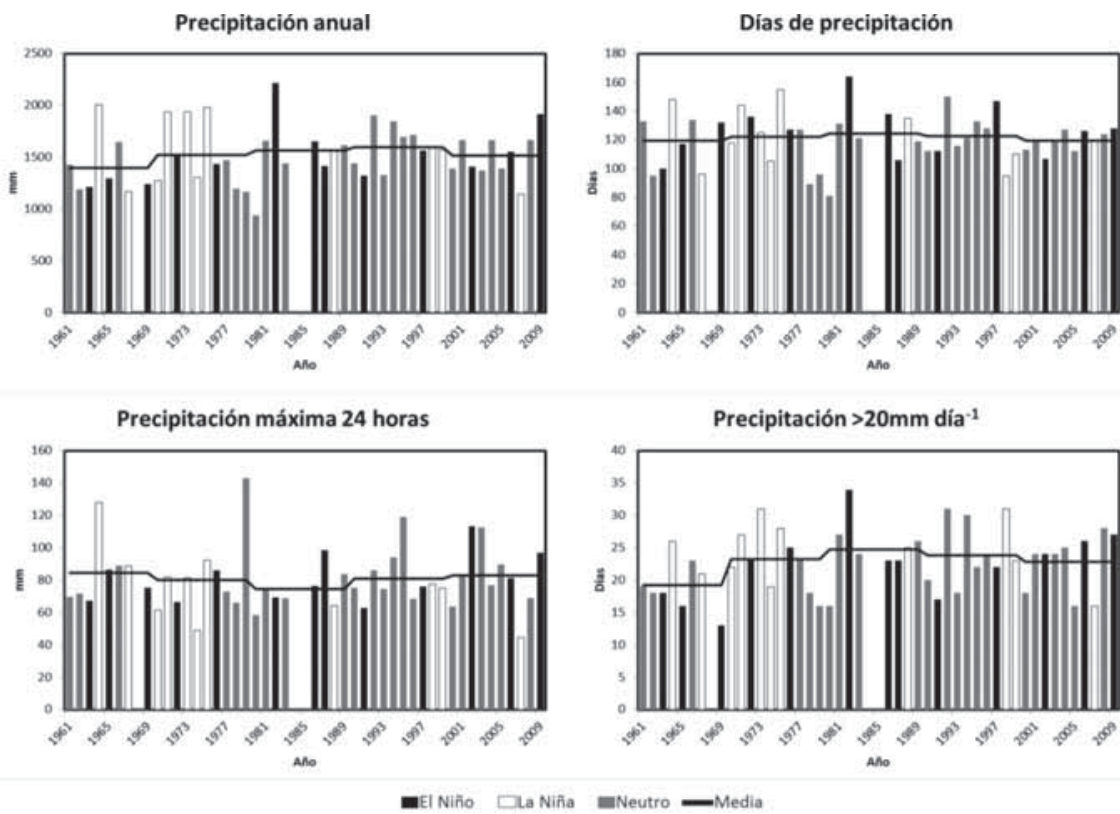

Fuente: elaboración propia a partir de datos del INMET y de COAPS.

CUAdro 1

COEFICIENTE DE CORRELACIÓN $(\mathrm{P}<0,05)$ ENTRE LOS DIFERENTES PARÁMETROS DE PRECIPITACIONES EN EL PERIODO 1961-2010

\begin{tabular}{lc}
\hline \multicolumn{1}{c}{ Parámetros } & $\mathrm{r}$ \\
\hline PPtot - Días & 0,77 \\
PPtot - PPmax & - \\
PPtot - >20 mm & 0,84 \\
Días - PPmax & - \\
Días - >20 mm & 0,52 \\
PPmax - >20 mm & - \\
\hline
\end{tabular}

Fuente: elaboración propia a partir de datos del INMET.

Estudios Geográficos, Vol. LXXIII, 272, pp. 325-331, enero-junio 2012

ISSN: 0014-1496, eISSN: 1988-8546, doi: 10.3989/estgeogr.201212 
Respecto del fenómeno ENOS, durante el periodo analizado ocurrieron 22 años de fase normal o neutra, 13 años de fase cálida o «El Niño», y 11 años en fase fría o «La Niña» (figura 2 y cuadro 2). A pesar de que la mayor precipitación se registró durante un año «El Niño»(1982), no es posible establecer una correlación significativa entre el aumento de lluvias y años de fase cálida, ni una disminución de precipitaciones en años «La Niña». El test de varianza no identifica diferencias estadísticas significativas entre los diferentes parámetros evaluados, afirmando que el fenómeno ENOS no ha influenciado el patrón de lluvias en el área de estudio.

\section{CUAdRo 2}

MEDIA DE LOS PARÁMETROS PLUVIOMÉTRICOS SEGÚN DÉCADA Y ENOS

\begin{tabular}{crcccc}
\hline \multicolumn{5}{c}{$(\mathbf{n}=$ años evaluados $)$} \\
\hline Década & $\mathbf{n}$ & PPtot $(\mathbf{m m})$ & Días/año & PPmax (mm) & $>20$ mm (días) \\
\hline 1960 & 8 & 1.398 & 119 & 84 & 19 \\
1970 & 10 & 1.522 & 122 & 80 & 23 \\
1980 & 8 & 1.562 & 124 & 74 & 25 \\
1990 & 10 & 1.596 & 122 & 81 & 24 \\
2000 & 10 & 1.516 & 120 & 83 & 23 \\
\hline ENOS & $\mathbf{n}$ & PPtot (mm) & Días/año & PPmax (mm) & $>20 \mathrm{~mm}$ (días) \\
\hline El Niño & 13 & 1.520 & 126 & 81 & 22 \\
La Niña & 11 & 1.588 & 122 & 76 & 24 \\
Neutro & 22 & 1.491 & 118 & 82 & 22 \\
\hline
\end{tabular}

Fuente: elaboración propia a partir de datos de INMET y de COAPS.

\section{DISCUSIÓN}

La ausencia de variaciones en el patrón de precipitaciones producidas por el cambio climático corrobora lo observado por Dufek y Ambrizzi (2008), donde sólo la mitad de 59 estaciones meteorológicas distribuidas en todo el estado de Sao Paulo mostró cambios significativos después de 49 años de mediciones. Los resultados de este artículo también concuerdan con un estudio realizado en 
Campinas-SP, a 130 km de São-Carlos-SP, donde se evaluaron las precipitaciones de 116 años sin encontrar efecto del cambio climático (Blain et al., 2007).

Respecto de los efectos del fenómeno ENOS en el área de estudio, los resultados de este trabajo no concuerdan con el realizado por Soler et al. (2010) en los municipios de Piracicaba-SP (años 1917-2002) y Ribeirão Preto-SP (años 1965-2002, a 65 y $85 \mathrm{~km}$ de São-Carlos-SP, respectivamente), donde se presentó una relación directa entre las precipitaciones y el fenómeno ENOS.

Con los datos pluviométricos de la estación climatológica principal de SãoCarlos-SP, es posible afirmar que existe relación entre la cantidad de precipitación anual, número de días lluviosos y la frecuencia de precipitaciones intensas ( $>20 \mathrm{~mm}$ dia-1). No se identificó efecto del cambio climático en el patrón de lluvias en las décadas entre 1961 y 2010, situación que confirma el comportamiento regional descrito en estudios anteriores. Respecto del fenómeno ENOS, no es posible distinguir su incidencia en el patrón pluviométrico anual como lo ha sido descrito para la región.

\section{AgRAdeCIMIENTOS}

A INMET por proveer los datos meteorológicos. A las agencias CNPQ e FAPESP por las becas de Iniciación Científica y Doctorado de los autores. El presente trabajo se encuadra en el proyecto de investigación "Modelaje de erosión de suelos y balance hídrico en cultivos de caña de azúcar y pradera en Latossolo" con financiamiento regular FAPESP 2010/00251-5.

\section{BIBLIOGRAFÍA}

Blain, G. C.; Piedade, S. M. D. S.; De Camargo, M. B. P. y Giarolla, A. (2007): "Distribuição temporal da precipitação pluvial mensal observada no posto meteorológico do Instituto Agronômico, em Campinas, SP". Bragantia, 66, pp. 347-355.

COAPS (2011): "ENSO Index According to JMA SSTA (1868-present)". Florida, Center for Ocean-Atmospheric Prediction Studies, The Florida State University. Disponible en: http://coaps.fsu.edu/jma.shtml. (Fecha de consulta: 17 de junio de 2011).

Dufek, A. S. y Ambrizzi, T. (2008): "Precipitation variability in São Paulo State, Brazil". Theoretical and Applied Climatology, 93, pp. 167-178.

IMPE (2011): "El Niño e La Niña. Centro de Previsão de Tempo e Estudos Climáticos". São José dos Campos, Instituto Nacional de Pesquisas Espaciais, Ministério da Ciência, tecnologia e Inovação do Brasil. Disponible en: http://enos.cptec. inpe.br/. (Fecha de consulta: 17 de junio de 2011). 
IPCC (2007): "Climate Change 2007: Synthesis Report. Contribution of Working Groups I, II and III to the Fourth Assessment Report of the Intergovernmental Panel on Climate Change". Switzerland, IPCC, Geneva, 104 pp.

Nunes, L. H. (2000): "Seasonal changes in the rainfall pattern in São Paulo State, Brazil", en Rosenzweig, C. y Basher, R. E. (eds.): Proceedings of First International Forum on Climate Prediction, Agriculture and Development. New York, IRI publication, pp. 129-133.

Soler, C. M. T.; Sentelhas, P. C. y Hoogenboom, G. (2010): "The impact of El Niño Southern Oscillation phases on off-season maize yield for a subtropical region of Brazil". International Journal of Climatology, 30, pp. 1056-1066.

\section{Cristian Youlton, Taís Shiratsubaki, Paulo Tarso Sanches de Oliveira y Edson Wendland}

Departamento de Hidráulica e Saneamento Escola de Engenharia de São Carlos Universidade de São Paulo (EESC/ USP) 